\title{
OPTIMISATION DES PRÉLÉVEMENTS PAR ACCUMULATION ET DOSAGE PAR COUPLAGE GC/MS DES COV PRESENTS DANS LES STOCKAGES D'ORDURES MENAGĖRES PREMIËRE APPROCHE DE COMPARAISON DE MÉTHODES ANALYTIQUES
}

\author{
Rodica Chiriac*, Ludovic Fine**, Jean-Louis Gass***, Jean Carre****. \\ * Doctorante Ademe, Laboratoire d'application de la chimie à l'environnement, \\ ** Technicien CNRS, Département Analyse de gaz, Service central d'analyse CNRS \\ *** Ingénieur CNRS, Responsable du Service analyse de gaz, Service central d'analyse CNRS, \\ ***** Maître de conférences, Laboratoire d'application de la chimie à l'environnement,
}

Le travail présenté constitue une approche de l'optimisation des conditions nécessaires à l'analyse des composés organiques volatils (COV) émis par des ordures ménagères en cours de maturation dans un pilote. Un volume connu de la phase gaz du pilote parcourt des tubes à accumulation de différentes natures : Tenax, Chromosorb, Tenax-carbone graphité et charbon actif. Les COV piégés sur les trois premiers types de tubes ont été extraits pas désorption thermique et par extraction au sulfure de carbone pour les tubes à charbon actif. Plus de 200 COV sont identifiés avec certitude et les performances des deux filières accumulation/désorption thermique et accumulation/extraction par solvant sont comparées. Quelques précautions à prendre pour un dosage quantitatif fiable sont émises.

The present article is devoted to an approach of optimization of required conditions to traces VOCs analysis coming from household wastes stocked in specially designed pilots for studying maturation. A known volume of the gas phase goes through tubes filled with various adsorbents like Tenax, Chromosorb, Tenaxgraphitized carbon and active charcoal. To extract VOCs, thermal desorption has been used for the first three adsorbents while CS2 extraction has been applied on active charcoal tubes. About 200 VOCs were surely identified and performances of the two sample preparation processes were compared : accumulation followed by thermal desorption or followed by solvent extraction. Both analysis techniques are compared in order to set all the precautions for a reliable quantitative analysis.

\section{INTRODUCTION}

Optimiser des conditions de prélèvements et comparer des méthodes d'analyse requièrent de disposer d'une source d'émission de COV assez riche du point de vue de la complexité du mélange et assez stable à l'échelle de la durée d'un prélèvement, surtout lorsque celui-ci implique une phase d'accumulation sur support adsorbant.

Le stockage d'ordures ménagères a déjà été étudié en tant que source d'émission de COV et la littérature mentionne une série de travaux[1] [2] qui amorcent de fait l'approche comparative de méthodes d'investigation reposant sur l'analyse de prélèvements issus d'une phase d'accumulation, même si les auteurs visaient en priorité une évaluation de l'impact de ces émissions sur l'environnement.

Par exemple, Allen et collaborateurs ${ }^{[I]}$ utilisent, sur site, le prélèvement par accumulation sur des tubes contenant un empilement de matériaux adsorbants (Tenax TA, Chromosorb 102 et Carbosieve SIII). La thermodésorption est conduite au laboratoire sur un ATD 50 Perkin Elmer couplé à un équipement GC/MS Hewlett Packard GC5890/MS5970. Ils détectent ainsi environ 140 composés.

Bradley et collaborateurs ${ }^{[2]}$ prélèvent d'abord dans des sacs Tedlar, ou des canisters, avant d'accumuler sur Tenax ou Chromosorb 102 au laboratoire, ou encore prélèvent sur site avec adsorption sur Tenax. Ils identifient ainsi environ 200 composés, également au moyen de la technique GC/MS.

Une étude bibliographique récente[3], employant un panel de techniques analytiques étendu, recense plus de 500 composés présents dans les gaz issus de décharges d'ordures ménagères. Ce résultat a conduit l'Agence de l'environnement anglaise[4] à proposer un suivi de molécules jugées prioritaires au moyen de la technique analytique suivante : adsorption dynamique sur une car- 
touche duale Tenax TA-Sphericarb, suivie d'une désorption thermique.

\section{MATÉRIEL ET MÉTHODES}

\section{Conditions expérimentales}

L'accès à des casiers pilotes de stockage d'ordures ménagères $(O M)$ de $8 \mathrm{~m}^{3}$ de volume nous a opportunément ouvert des possibilités de mesures conduites sur la phase gaz qui confirment la richesse déjà mentionnée. Cette richesse se traduit à la fois par le nombre de composés émis, mais aussi par l'étendue de la gamme des concentrations rencontrées. Ce dernier point posera des problèmes en approche quantitative, comme nous le verrons plus tard, mais en revanche, nous permet de détecter et identifier un maximum de composés, y compris ceux présents habituellement à l'état de traces. Par ailleurs le volume d'OM stockées nous assure une cinétique de maturation assez lente pour admettre que cette composition demeure qualitativement et quantitativement constante dans les conditions opératoires requises pour une phase d'accumulation.

Ces déchets ménagers proviennent d'un centre d'enfouissement technique d'OM avec la même composition reconstituée (déchets fins et déchets volumineux) que pour un envoi en alvéole de stockage. Le compactage a été effectué par damage en essayant d'imiter au maximum les conditions sur site.

Les prélèvements ayant eu lieu en juin avec une température de $28^{\circ} \mathrm{C}$, il paraît raisonnable d'envisager une température équivalente sur le point de prélèvement, la canne à gaz métallique utilisée homogénéisant la température sur toute sa longueur par conduction naturelle. La pression peut être assimilée à la pression atmosphérique, car la dépression est minime, à cause du faible débit gazeux de prélèvement.

Nous avons pu tester différents matériaux adsorbants et différentes méthodes analytiques, thermodésorption ou extraction par solvant, qui ouvrent la voie à des tests plus systématiques qui permettront à terme d'argumenter le choix de la méthode en fonction de critères qui peuvent être très variables : rapidité d'établissement de diagnostic, portabilité de la méthode jusque sur le site d'émission, niveau d'exhaustivité recherché, etc.

En effet, même s'il existe une norme ${ }^{[5]}$ pour le prélèvement par accumulation suivi de la désorption thermique, l'analyse d'un mélange aussi complexe que celui étudié ici demande d'optimiser les conditions opératoires, sans chercher à occulter les limites de la technique et en dégageant les risques d'erreurs ou d'interférences entre produits.

\section{Techniques de prélèvement}

Les prélèvements ont été réalisés sur la phase gazeuse en équilibre avec le déchet solide, au sein de ce dernier pour les raisons suivantes:
- l'atmosphère d'un casier peut être "confinée" avec un maximum de précautions, et les émissions sont alors soigneusement collectées et canalisées. Un casier peut également être ouvert à l'atmosphère, sans collecte du biogaz. Dans tous les cas il nous paraît préférable de conduire le prélèvement au sein du tas afin de s'affranchir de toutes fuites ou contaminations extérieures au milieu réactionnel ;

- la mise en œuvre est relativement aisée (implantation d'une canne à gaz réalisée au laboratoire) ;

- enfin, pour ce qui a trait au suivi de la maturation du tas, l'information la plus pertinente provient de l'identification et la quantification des espèces émises en son sein.

L'humidité relative, souvent proche de la saturation dans ce milieu, génère une des difficultés à maîtriser : le retour à la température ambiante du gaz prélevé provoque la condensation et peut noyer l'adsorbant au cours de la phase d'accumulation. L'utilisation d'une canne métallique de section suffisante et fichée verticalement dans le tas est un moyen de prévention satisfaisant si le débit volumique prélevé demeure faible, ce qui est le cas ici.

Plusieurs procédures de prélèvement avec accumulation étaient possibles :

- Ire solution : le débit volumique disponible au moyen de la canne était suffisant pour alimenter plusieurs pompes volumiques montées en parallèle. Cette solution, idéale, n'a pas pu être mise en œuvre pour des raisons essentiellement matérielles : il faut mettre en place des raccords de connexion et disposer d'un nombre de pompes suffisant au même moment, le tout au dessus d'un tas d'ordures ménagères.

- 2e solution : une seule pompe volumique, plus performante, assure le gonflage d'un sac Tedlar de 10 litres, ce qui fournit une réserve de mélange gazeux à analyser, à la pression atmosphérique. L'inconvénient majeur de cette technique est la vapeur d'eau qui condense dans le sac, ce phénomène pouvant altérer sérieusement les résultats analytiques qui en résultent. Cette technique de prélèvement aurait été acceptable si le seul objectif de ces mesures avait été une étude comparative de méthodes analytiques. En effet, dans les minutes qui suivent, une pompe à débit contrôlé prélève un volume connu qui a été contraint de transiter dans un tube adsorbant. Ainsi, tous les tubes destinés à l'étude comparative proviennent d'un seul prélèvement stocké dans le sac Tedlar initial. Mais, le premier objectif de l'étude demeure la validité, au minimum qualitative, de résultats analytiques et en conséquence cette technique de prélèvement a été écartée.

- 3e solution, un compromis : les prélèvements ont été conduits en série, à partir de la même canne métallique, au moyen de deux types de pompes aspirantes, leur débit étant vérifié dans les conditions opératoires, à l'aide d'un débitmètre à bulles. 
Il est connu que les conditions de non-perçage du matériau adsorbant dépendent de la nature de ce dernier, du débit gazeux de la concentration et du nombre des COV qui doivent être fixés. Les tubes à charbon actif devant subir une extraction par solvant présentent une partie aval, utilisable pour un test permettant la vérification du non-perçage. En revanche, les tubes subissant une désorption thermique sont dépourvus de cette possibilité ce qui impose soit un montage de deux tubes en série, montage recommandé, soit des prélèvements à volumes aspirés croissants permettant d'évaluer la limite supérieure du volume admissible.

\section{Procédure analytique}

La technique analytique reconnue est basée sur la chromatographie en phase gazeuse couplée à un spectromètre de masse qui assure l'identification des COV et parfois autorise une déconvolution lorsque la séparation issue de la colonne est incomplète.

En effet, les COV issus de déchets d'OM constituent un mélange complexe contenant plus d'une centaine de composants, distribués dans plusieurs familles chimiques, avec une gamme de masse très étendue allant des produits légers, incondensables dans les conditions ordinaires comme le chlorure de vinyle, jusqu'aux produits semi-volatils comme le naphtalène. II parait donc illusoire de prétendre avoir réussi la fixation de ces composés sur un seul support adsorbant, comme il est hautement probable que la colonne du chromatographe, aussi performante soit-elle, peinera à assurer une séparation totale. Le choix de conditions de prélèvement et d'analyse correspondra donc toujours à un compromis.

Nous avons donc tenté une première approche de comparaison de performances portant à la fois sur des adsorbants, et sur des méthodes d'extraction, tout en mobilisant différents types d'équipements analytiques : - un couplage GC5890/MS5973 Agilent Technologies pour les analyses après extraction par solvant. Le GC est équipé d'une colonne J\&W DB-5MS (longueur : 60 mètres, diamètre : $0,257 \mathrm{~mm}$, phase stationnaire : copolymère de diphényl (5\%) et diméthyl ( $95 \%$ ) siloxane). Le montage d'une colonne aussi performante a été effectué dans le but d'avoir une efficacité analytique maximale pour un usage polyvalent de l'appareil ;

- un couplage thermodésorbeur Ultra Unity Markes/GC6890N/MS5973 Agilent Technologies pour les analyses après désorption thermique. Ce thermodésorbeur est également un passeur automatique d'une capacité de 100 tubes. Le GC est équipé de la colonne « constructeur » initiale, HP5MS, $30 \mathrm{~m}, 0,25 \mathrm{~mm}, 25 \mu \mathrm{m}$ d'épaisseur de phase, livrée avec tous les appareils de séries 5890 et 6890 . Le travail présenté ici montre que cette colonne assure une séparation suffisante pour un nombre très important de COV.

Les résultats présentés ci-après ont été acquis sur un prélèvement effectué à $60 \mathrm{~cm}$ de profondeur dans un casier pilote "ouvert", d'une contenance de $8 \mathrm{~m}^{3}$, rempli de déchets ménagers ayant une vingtaine de jours de stockage.

Quelle que soit la nature de l'adsorbant destiné à la fixation des COV, des conditions identiques d'aspiration ont été imposées : un temps d'aspiration de 15 minutes avec un débit de $130 \mathrm{ml} / \mathrm{min}$. Le choix de ce débit un peu élevé a été volontaire pour deux raisons : avoir une durée totale de prélèvement limitée à une heure pour l'ensemble des quatre tubes et avoir une dépression suffisante pour bien capter les COV présents dans la matrice déchets.

Nous avons testé trois types de cartouches à accumulation Markes pour la méthode relative à la thermodésorption (Tenax, chromosorb, Tenax/carbone graphité) et des cartouches SKC à charbon actif destinées à l'extraction par solvant.

Nous avons donc effectué quatre prélèvements différents, dans les mêmes conditions, dans la même heure pour charbon actif, Tenax, Chromosorb, Tenax-carbone graphité.

\section{Conditions analytiques relatives à l'extraction par sol-} vant

Les produits piégés sur charbon actif sont extraits par une sonication de 14 minutes avec du sulfure de carbone Fluka référence 84713 (exempt de benzène) selon la norme NF ISO I6200-I[6]. Une addition de $21,4 \mathrm{mg} . \mathrm{L}^{-1}$ de bromododécane choisi comme étalon interne permet si besoin une analyse quantitative pour les produits retenus.

Les conditions analytiques sont les suivantes : injection en mode splitless et au terme d'un palier de 2 minutes à $36^{\circ} \mathrm{C}$, la température de la colonne est augmentée à raison de $5^{\circ} \mathrm{C} /$ minute jusqu'à $260^{\circ} \mathrm{C}$. L'alimentation du filament est coupée pendant 12 minutes (élution du solvant en mode splitless).

\section{Conditions analytiques relatives à la thermodésorp- tion}

Les produits piégés sont d'abord désorbés des tubes à $280^{\circ} \mathrm{C}$ pour les tubes Tenax et Tenax-carbone graphité et à $250^{\circ} \mathrm{C}$ pour les tubes Chromosorb. Les produits élués sont condensés dans un piège à $0^{\circ} \mathrm{C}$ et envoyés sur le GC/MS par chauffage du piège à $300^{\circ} \mathrm{C}$.

Les conditions analytiques sont alors les suivantes : isotherme d'une durée de 2 minutes à $35^{\circ} \mathrm{C}$, suivie d'une première rampe de montée en température à $5^{\circ} \mathrm{C}$ /minute jusqu'à $220^{\circ} \mathrm{C}$, puis d'une deuxième rampe de montée en température à $15^{\circ} /$ minute de $220^{\circ} \mathrm{C}$ jusqu'à $300^{\circ} \mathrm{C}$, et enfin d'un palier isotherme de 2 minutes à $300^{\circ} \mathrm{C}$.

\section{Critères d'identification définis pour la thermodé- sorption}

La procédure d'identification est classique : comparaison du spectre obtenu en mode SCAN avec ceux rassem- 
blés dans la bibliothèque de spectres NIST. Les résultats obtenus sont rassemblés dans le tableau $\mathrm{I}$.

Ce tableau ne comporte que les composés dont l'identification paraît certaine : nous avons d'abord retenu les composés identifiés avec un facteur de ressemblance supérieur à 90/100 (numérotés en gras), puis ceux dont le facteur de ressemblance est supérieur à 75 .

En ce qui concerne les composés identifiés avec un facteur de ressemblance inférieur à 75 , ne sont retenus que ceux identifiés à partir de tubes "Markes" dont l'adsorbant est différent, mais dont le temps de rétention est identique. Le recoupement de ces deux critères (nature de l'adsorbant et temps de rétention identique) permet de reconnaître pratiquement la totalité des composés qui ont été séparés lors des analyses avec un degré de sûreté d'identification tout à fait satisfaisant.

La conséquence de cette rigueur dans le choix des critères d'identification peut avoir pour conséquence le rejet de COV alors qu'ils ont été séparés par chromatographie. Par exemple 206 pics, donc 206 composés au minimum, ont été détectés (mais pour certains, non identifiés au sens des critères définis ci-dessus) à partir de Tenax, 199 à partir de Chromosorb, et 191 à partir de Tenax-carbone graphité. La même démarche a été appliquée au charbon actif ce qui conduit à retenir 70 COV alors que 178 composés ont été détectés.

Pour assurer une lisibilité maximale du tableau, nous avons calculé un temps de rétention moyen, arrondi au centième de minute, pour ce qui concerne les trois analyses issues de la thermodésorption. L'écart entre les trois temps de rétention n'excède jamais deux centièmes de minute.

Quelques composés, en général majoritaires, sont identifiés à partir des trois tubes, avec des facteurs de ressemblance supérieurs à 90 . Ces composés sont signalés par des chiffres en caractères gras (facteurs de ressemblance et surfaces des pics).

Afin d'éviter la surcharge des tableaux nous avons volontairement omis la mention du $\mathrm{N}^{\circ} \mathrm{CAS}$ malgré l'intérêt de cette information. En effet, des synonymes existent pour de nombreux produits. Nous avons retenu pour la plupart, l'écriture conforme aux normes, sauf quand la longueur de l'appellation était incompatible avec l'espace alloué dans le tableau $I$.

(junipène au lieu de IS- $(I \alpha, 3 a \beta, 4 \alpha, 8 a \beta)]$ décahydro4,8,8-triméthyl-9-méthylène- I,4-Méthanoazulène).

\section{RÉSULTATS ET COMMENTAIRES}

Ce commentaire de résultats porte, dans un premier temps, sur les performances de la thermodésorption en général et ce que peut apporter l'emploi d'adsorbants réputés plus spécifiques de certains composés ou familles de composés.

Rappel sur les tubes utilisés : Tenax, Chromosorb, Tenax-carbone graphité.

\begin{tabular}{|c|c|c|c|c|c|c|c|c|}
\hline & & $\begin{array}{r}\text { au I : Résulta } \\
\text { therm }\end{array}$ & & C & & & & \\
\hline $\begin{array}{l}N \\
\text { du } \\
\text { pic }\end{array}$ & $\begin{array}{l}\text { TR } \\
\text { min. }\end{array}$ & Produit & Ten & $\operatorname{nax} C$ & trome & osorb & & $\begin{array}{l}\text { enax } \\
\text { bone } \\
\text { phité }\end{array}$ \\
\hline & & & Q & S.107 & $\mathrm{Q}$ & S. $10^{7}$ & $\mathrm{Q}$ & S. $10^{7}$ \\
\hline I & 1,81 & éthanol & 91 & 1,66 & - & - & 91 & 2,2 \\
\hline 2 & 1,86 & 2-méthyl butane & 91 & 5,55 & 55 & 247,6 & & 17,16 \\
\hline 3 & 1,89 & I-pentène & - & - & 85 & 65,9 & - & - \\
\hline 4 & 1,91 & pentane & - & - & 86 & 72 & - & - \\
\hline 5 & 1,93 & alcool isopropylique & 87 & 8,77 & 35 & 17,0 & 64 & 12,52 \\
\hline 6 & 2,02 & diméthylsulfure & 94 & 0,52 & - & - & 94 & 0,56 \\
\hline 7 & 2,04 & I,1,2-trichloroéthane & - & - & 91 & 3,03 & - & - \\
\hline 8 & 2,06 & 2,2-diméthyl butane & - & - & 83 & 5,41 & - & - \\
\hline 9 & 2,07 & acétate de méthyle & 78 & 2,92 & - & - & 59 & 2,69 \\
\hline 10 & 2,25 & I-propanol & 91 & 52,2 & 72 & 39,4 & 87 & 78,0 \\
\hline II & 2,35 & I-propanol & - & - & 90 & 97,1 & - & - \\
\hline 12 & 2,36 & 3-méthylpentane & 72 & 0,887 & - & - & 86 & 2,17 \\
\hline 13 & 2,43 & 2-méthyl I-pentène & 60 & $\mathrm{I}, 14$ & - & - & 91 & 2,44 \\
\hline 14 & 2,51 & 2-butanol & 47 & 61,3 & & & 43 & 50,44 \\
\hline 15 & 2,57 & 2-butanol & - & - & 83 & 28 & - & - \\
\hline 16 & 2,65 & 2-butanol & - & - & 83 & 103,6 & - & - \\
\hline 17 & 2,72 & acétate d'éthyle & - & - & 72 & 160,4 & 35 & 37,9 \\
\hline 18 & 2,79 & méthylcyclopentane & - & - & - & - & 87 & 2,25 \\
\hline 19 & 2,88 & propanoate de méthyle & 72 & 29,1 & 90 & 12,9 & 49 & 29,77 \\
\hline 20 & 2,98 & 2-méthyl I-propanol & - & - & 91 & 17,9 & - & - \\
\hline 21 & 3,09 & I,2-dichloroéthane & 96 & 1,55 & - & - & - & - \\
\hline 22 & 3,11 & 3,3-diméthyl pentane & - & - & 50 & $\mathrm{I}, 40$ & 72 & 1,05 \\
\hline 23 & 3,18 & 3-méthyl butanal & - & - & 64 & 0,611 & 58 & 0,39 \\
\hline 24 & 3,26 & benzène & 91 & 31,4 & 91 & 36,0 & 94 & 34,0 \\
\hline 25 & 3,38 & I-butanol & - & - & 90 & 28,9 & - & - \\
\hline 26 & 3,45 & I-butanol & 78 & 156 & - & - & 83 & 113,5 \\
\hline 27 & 3,58 & I-butanol & 80 & 165,1 & - & - & - & - \\
\hline 28 & 3,70 & I-butanol & - & - & 90 & 259,8 & 80 & 253,5 \\
\hline 29 & 3,73 & méthyl isobutanoate & - & - & 62 & 3,35 & 49 & 5,89 \\
\hline 30 & 3,80 & 3-méthyl 2-butanol & - & - & - & - & 80 & 0,85 \\
\hline 31 & 3,85 & allyl méthyl sulfure & 94 & 2,89 & 45 & 5,33 & 90 & 5,62 \\
\hline 32 & 3,91 & trichloréthylène & 96 & 29,7 & 93 & 16,0 & 97 & 34,1 \\
\hline 33 & 4,06 & 2-pentanol & 78 & 8,8 & - & - & - & - \\
\hline 34 & 4,15 & 2-pentanol & - & - & 72 & 5,90 & 59 & 12,0 \\
\hline 35 & 4,30 & propanoate d'éthyle & 83 & $|3|$ & - & - & 83 & 134,5 \\
\hline 36 & 4,34 & acétate de propyle & 83 & 18,2 & 78 & 11,2 & 64 & 18,8 \\
\hline 37 & 4,41 & méthyl cyclohexane & 91 & 2,50 & 91 & 7,57 & 94 & 10,35 \\
\hline 38 & 4,52 & butanoate de méthyle & 94 & 64,6 & 94 & $4 I, I$ & 94 & 65,8 \\
\hline 39 & 4,61 & 2,5-diméthyl hexane & - & - & - & - & 87 & 0,7 \\
\hline 40 & 4,67 & éthyl cyclopentane & 64 & 2,21 & 45 & 2,37 & - & - \\
\hline 41 & 4,75 & isobuténylcarbinol & 93 & 0,83 & - & - & - & - \\
\hline 42 & 4,78 & 3-méthyl I-butanol & 90 & 3,45 & 59 & 5,98 & 59 & 5,35 \\
\hline 43 & 4,98 & diméthyldisulfure & 96 & 91,2 & 96 & 142,2 & 96 & 80,2 \\
\hline 44 & 5,01 & 2-méthyl I-butanol & 83 & 11,6 & 83 & 13,9 & 64 & 9,03 \\
\hline 45 & 5,15 & 2,3,5-triméthyl pentane & - & - & 64 & 1,86 & 90 & 1,79 \\
\hline 46 & 5,33 & propionate d'isopropyle & 90 & 2,89 & 50 & 2,46 & 59 & 3,71 \\
\hline 47 & 5,46 & éthyl isobutanoate & 58 & 33,1 & 49 & 21,6 & 46 & 36,2 \\
\hline 48 & 5,57 & toluène & 91 & 99,4 & 94 & 66,6 & 91 & 104 \\
\hline 49 & 5,64 & I-pentanol & 64 & $|, 5|$ & - & - & 86 & 2,57 \\
\hline
\end{tabular}




\begin{tabular}{|c|c|c|c|c|c|c|c|c|}
\hline & & $\begin{array}{r}\text { tau I : Résultat } \\
\text { thermique }\end{array}$ & & 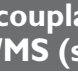 & & & & \\
\hline $\begin{array}{l}\mathrm{N} \\
\mathrm{du} \\
\text { pic }\end{array}$ & $\begin{array}{c}\text { TR } \\
\text { min. }\end{array}$ & Produit & Ten & $\operatorname{lax}$ & irom & osorb & & $\begin{array}{l}\text { nax } \\
\text { bone } \\
\text { phité }\end{array}$ \\
\hline & & & $\mathrm{Q}$ & S.107 & Q & S.107 & Q & S. $10^{7}$ \\
\hline 50 & 5,72 & 3-méthylheptane & 90 & 1,54 & - & - & 90 & 2,92 \\
\hline 51 & 5,90 & I-pentanol & 80 & 33,8 & 90 & 40,2 & 86 & 43,8 \\
\hline 52 & 6,26 & |-octène & 93 & 2,22 & 73 & 2,22 & 93 & 2,96 \\
\hline 53 & 6,37 & I,2-diméthylcyclohexane & - & - & 83 & 1,46 & 93 & 2,1 \\
\hline 54 & 6,52 & octane & 95 & 16,3 & 95 & 11,6 & 91 & 17,7 \\
\hline 55 & 6,82 & butanoate d'éthyle & 90 & 561 & 96 & 360,1 & 91 & 585 \\
\hline 56 & 7,00 & propanoate de propyle & 83 & 27,5 & 83 & 16,0 & 83 & 29,0 \\
\hline 57 & 7,12 & acétate de butyle & 83 & 20,5 & 83 & 13,9 & 83 & 22,4 \\
\hline 58 & 7,26 & 2,4-diméthylheptane & 95 & 3,58 & 95 & 3,96 & 95 & 7,3 \\
\hline 59 & 7,38 & hexaméthyltrisiloxane & - & - & - & - & 90 & 4,36 \\
\hline 60 & 7,41 & pentanoate de méthyle & 93 & 9,48 & 87 & 5,77 & 87 & 12,4 \\
\hline 61 & 7,56 & éthylcyclohexane & 87 & 3,27 & 91 & 1,86 & 91 & 4,76 \\
\hline 62 & 7,671 & I, I,3-triméthylcyclohexan & e46 & 0,93 & 60 & 3,45 & 87 & 8,60 \\
\hline 63 & 7,85 & 4-méthyl-I-pentanol & 78 & 5,67 & - & - & - & - \\
\hline 64 & 7,86 & diméthylsulfoxyde & - & - & 93 & 16,2 & - & - \\
\hline 65 & 7,98 & chlorobenzène & - & - & 95 & 23,1 & - & - \\
\hline 66 & 8,00 & butanoate de Iméthyléthyle & 58 & 17,1 & - & - & 60 & 19,2 \\
\hline 67 & 8,16 & I,3,5-triméthylcyclohexane & 91 & 0,833 & 91 & 9,61 & 91 & 3,72 \\
\hline 68 & $8,30 t$ & butanoate de 2 méthyléthyle & 43 & 37,6 & 58 & 35,4 & 43 & 40,2 \\
\hline 69 & 8,39 & heptanoate d'éthyle & 35 & 19,1 & 59 & 23,3 & 45 & 21,3 \\
\hline 70 & 8,55 & éthylbenzène & 91 & 28,2 & 91 & 33,9 & 91 & 34,3 \\
\hline 71 & 8,63 & 4-méthyloctane & 93 & 7,15 & 90 & 4,71 & 87 & 9,31 \\
\hline 72 & 8,64 & 2,6-diméthylundécane & - & - & 83 & 6,87 & - & - \\
\hline 73 & 8,82 & p.xylène & 97 & 62,6 & 97 & 47,6 & 97 & 66,5 \\
\hline 74 & 8,89 & 3-méthyloctane & - & - & 53 & 5,51 & 64 & 6,06 \\
\hline 75 & 8,91 & diméthylsulfoxyde & - & - & 94 & 4,71 & - & - \\
\hline 76 & 8,97 & I-hexanol & 78 & 23,9 & 72 & 24,8 & 64 & 22,8 \\
\hline 77 & 9,11 & 1,2,4-triméthylcyclohexane & 93 & 0,838 & 64 & 0,80 & 87 & 3,67 \\
\hline 78 & 9,17 & $\begin{array}{l}\text { acétate de 3méthyl } \\
\text { I-butanol }\end{array}$ & 83 & 2,18 & - & - & - & - \\
\hline 79 & 9,40 & $\begin{array}{l}\text { I-éthyl 4-méthyl } \\
\text { cyclohexane }\end{array}$ & 91 & 3,78 & 91 & 5,67 & 91 & 7,49 \\
\hline 80 & 9,46 & $\begin{array}{l}\text { I-éthyl 2-méthyl } \\
\text { cyclohexane }\end{array}$ & 86 & 2,12 & 70 & 3,28 & 87 & 3,60 \\
\hline 81 & 9,54 & styrène & 97 & 17,7 & 95 & 13,9 & 95 & 19,6 \\
\hline 82 & 9,62 & p xylène & 91 & 25,7 & 91 & 16,5 & 91 & 27,1 \\
\hline 83 & 9,93 & butanoate de propyle & 91 & 72,1 & 64 & 39,3 & 87 & 75,8 \\
\hline 84 & 9,97 & butanoate de butyle & 64 & 55,2 & 74 & 54,3 & 83 & 63,8 \\
\hline 85 & 10,04 & octanoate d'éthyle & 32 & 45,9 & 37 & 34,3 & 35 & 52,3 \\
\hline 86 & 10,17 & $\begin{array}{l}\text { I-éthyl 4-méthyl } \\
\text { cyclohexane }\end{array}$ & 93 & 1,025 & 83 & 1,89 & 91 & 2,47 \\
\hline 87 & 10,29 & propanoate de butyle & 83 & 20,4 & 83 & 16,7 & 83 & 21,9 \\
\hline 88 & 10,60 & $\begin{array}{c}\text { I, I,7-triméthyl-tricyclo } \\
{[2.2 .1 .02,6] \text { heptane }}\end{array}$ & 38 & 0,910 & 94 & 2,74 & 94 & 3,94 \\
\hline 89 & 10,63 & diméthylsulfone & 91 & 1,58 & - & - & - & - \\
\hline 90 & 10,74 & I-méthyléthylbenzène & 91 & 11,5 & 87 & 8,75 & 91 & 11,65 \\
\hline 91 & 10,90 & propylcyclohexane & 91 & 8,84 & 74 & 7,07 & 83 & 10,3 \\
\hline 92 & 11,08 & alpha pinène & 95 & 45,7 & 94 & 84,0 & 94 & 125,6 \\
\hline 93 & 11,35 & $\begin{array}{c}\text { butanoate } \\
\text { de méthylhexyle }\end{array}$ & 39 & 87,5 & 45 & 65,6 & 42 & 98,3 \\
\hline
\end{tabular}

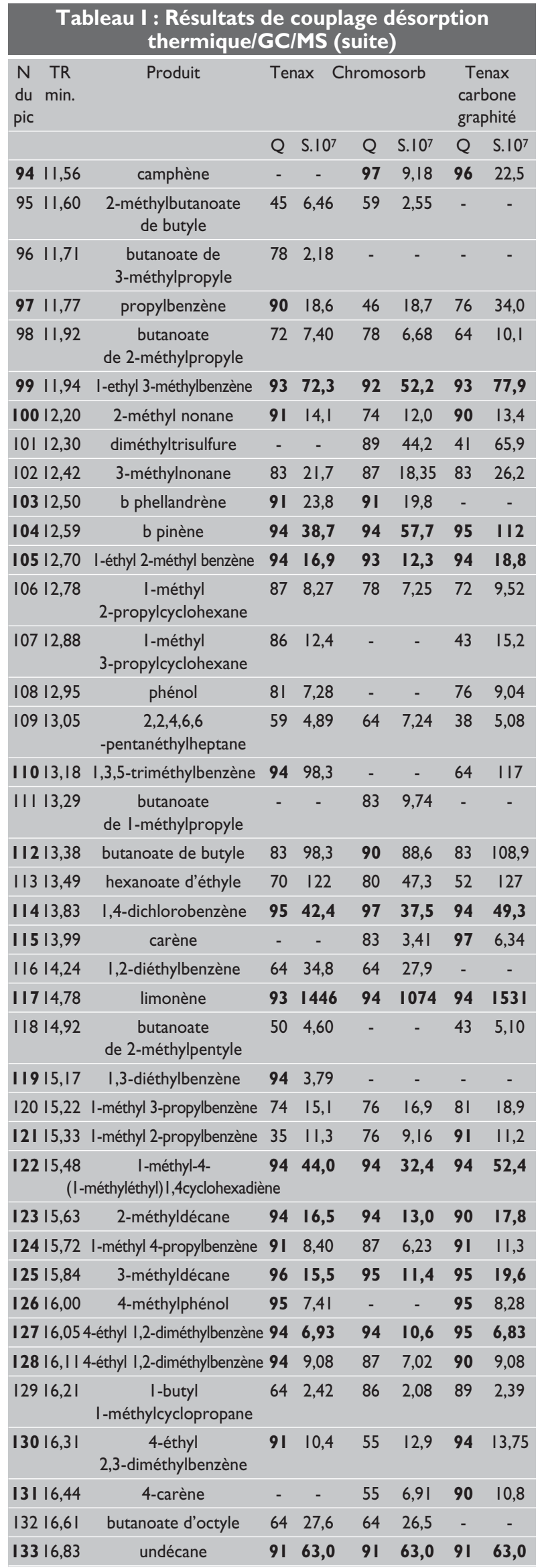




\begin{tabular}{|c|c|c|c|c|c|c|c|}
\hline & $\begin{array}{r}\text { leau I : Résultats } \\
\text { thermique }\end{array}$ & & 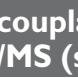 & & & & \\
\hline $\begin{array}{ll}N & T R \\
\text { du } & \text { min. } \\
\text { pic } & \end{array}$ & produit & Ten & $\operatorname{nax}$ & romo & ssorb & & $\begin{array}{l}\text { nax } \\
\text { pone } \\
\text { phité }\end{array}$ \\
\hline & & Q & S. $10^{7}$ & Q & S. $10^{7}$ & $\mathrm{Q}$ & S. $10^{7}$ \\
\hline$|34| 7,02$ & $\begin{array}{l}\text { 4-méthyl-I-(I-méthyléthyl) } \\
\text {-bicyclo[3.I.0]hexan-3-one }\end{array}$ & 97 & 20,2 & 97 & 15,4 & 96 & 19,5 \\
\hline $135 \mid 7,12$ & 2-méthyl trans décaline & 97 & 5,28 & 97 & 3,97 & 97 & 4,61 \\
\hline 13617,19 & 2,6-diméthyldécane & 50 & 3,44 & 53 & 2,31 & - & - \\
\hline $137 \mid 7,25$ & diéthénylbenzène & - & - & 87 & 3,94 & - & - \\
\hline |38 |7,45 & I,2,3,4-tétraméthyl benzène & - & - & 93 & 4,17 & 94 & 5,54 \\
\hline |39|7,49 & $\begin{array}{c}\text { trans-p-2,8- } \\
\text { menthadiène- I-ol }\end{array}$ & 93 & 22,6 & - & - & - & - \\
\hline$|40| 7,66$ & 3,7-diméthyldécane & 70 & 8,78 & - & - & 92 & 7,43 \\
\hline$|4| 17,73$ & I-dodécène & 45 & 1,41 & 42 & 1,19 & 30 & 1,37 \\
\hline 14217,83 & $\begin{array}{c}\text { hexanoate } \\
\text { de 2-méthylpropyle }\end{array}$ & 42 & 4,10 & 45 & 3,87 & 50 & 4,55 \\
\hline 14317,89 & butylcyclohexane & 59 & 5,94 & 59 & 5,26 & 59 & 6,60 \\
\hline$|44| 8,03$ & hexylcyclopentane & 55 & 4,95 & 46 & 4,25 & 46 & 6,00 \\
\hline $145 \mid 8,20$ & I,4-diéthy|2méthy|benzène & 83 & 1,97 & 64 & 1,67 & 70 & 2,70 \\
\hline $146 \mid 8,26$ & camphor(D) & 91 & 3,25 & 70 & 2,97 & 83 & 3,09 \\
\hline$|47| 8,48$ & $\begin{array}{c}\text { I-éthyl } \\
\text { 3,5-diméthylbenzène }\end{array}$ & 58 & 5,35 & 49 & 4,16 & - & - \\
\hline$|48| 8,52$ & 3-éthylheptane & 11 & 3,53 & 30 & 2,09 & - & - \\
\hline $149 \mid 8,62$ & $\begin{array}{c}\text { benzoate de } \\
\text { 2-[(triméthylsilyl)oxy]-, } \\
\text { triméthylsilyle }\end{array}$ & 72 & 44,6 & 72 & 24,3 & 72 & 56,1 \\
\hline 15018,68 & 4-méthylundecane & 81 & 44,3 & 93 & 2,87 & 93 & 4,50 \\
\hline $15 \mid 18,82$ & 2-méthyldodécane & 76 & 7,35 & 58 & 6,29 & 64 & 7,77 \\
\hline 15218,89 & I,I-diméthylpropylbenzène & 46 & 3,27 & 43 & 3,72 & 38 & 4,38 \\
\hline 15319,02 & 3,8-diméthyldécane & 64 & 7,63 & 64 & 5,67 & 59 & 6,74 \\
\hline$|54| 9,3 \mid$ & $\begin{array}{l}\text { 4-méthyl-I- } \\
\text { (I-méthyléthyl)- } \\
\text { 3-cyclohexène-I-ol }\end{array}$ & 90 & 2,45 & 64 & 2,11 & 91 & 2,19 \\
\hline $155 \mid 9,52$ & $\begin{array}{c}\text { I-méthyl } \\
\text { 2-propylcyclohexane }\end{array}$ & 49 & 4,67 & 50 & 2,13 & 53 & 4,96 \\
\hline 15619,62 & $\begin{array}{l}\text { 2-méthylène-5- } \\
\text { (I-méthyléthényl)- } \\
\text { cyclohexanol }\end{array}$ & 87 & 4,72 & - & - & - & - \\
\hline $157 \mid 9,68$ & hexanoate de butyle & 74 & 8,71 & 78 & 10,8 & 64 & 10,58 \\
\hline $158 \mid 9,88$ & octanoate d'éthyle & 87 & 5,48 & 86 & 4,05 & 86 & 6,08 \\
\hline 15919,96 & dodécane & 93 & 19,7 & 94 & 10,5 & 94 & 20,0 \\
\hline 16020,37 & 2,6-diméthylundécane & 94 & 4,53 & 93 & 2,83 & 91 & 4,48 \\
\hline $16 \mid 20,50$ & diméthyltétrasulfure & 87 & 2,33 & - & - & 83 & 2,55 \\
\hline 16220,60 & $\begin{array}{l}\text { 2-méthyl-5- } \\
\text { (I-méhyléthényl)- } \\
\text { 2-cyclohexène-I-ol }\end{array}$ & 83 & 3,20 & - & - & - & - \\
\hline 16320,65 & $\begin{array}{c}\text { 2-butyl } \\
\text { I,I,3-triméthylcyclohexane }\end{array}$ & - & - & 50 & 0,54 & 52 & 0,73 \\
\hline 16421,13 & hexylcyclohexane & 72 & 1,07 & 87 & 0,976 & 83 & 0,98 \\
\hline 16521,22 & décylcyclopentane & - & - & - & - & 87 & 0,55 \\
\hline 16621,36 & d-carvone & 96 & 98 & 93 & 1,06 & 97 & 0,86 \\
\hline 16721,52 & 2,6-diméthylundécane & 58 & 0,80 & - & - & 76 & 1,54 \\
\hline 16821,65 & butyrate de linalyle & 90 & 0,62 & 83 & 0,64 & 90 & 0,90 \\
\hline $16921,7 \mid$ & 4-méthyldodécane & 96 & 0,63 & - & - & 95 & 0,62 \\
\hline 17022,12 & 2,6-diméthylheptadécane & 83 & 1,05 & 87 & 0,59 & 83 & 1,04 \\
\hline $17 \mid 22,35$ & docosane & 72 & 0,74 & - & - & 86 & 0,60 \\
\hline
\end{tabular}

\begin{tabular}{|c|c|c|c|c|c|c|c|}
\hline \multirow{3}{*}{$\begin{array}{ll}N & T R \\
\text { du } & \text { min. } \\
\text { pic } & \end{array}$} & $\begin{array}{l}\text { lu I : Résulta } \\
\text { thermiq }\end{array}$ & & & & & & \\
\hline & \multirow{2}{*}{ therm } & \multicolumn{2}{|c|}{ Tenax } & \multicolumn{2}{|c|}{ Chromosorb } & \multicolumn{2}{|c|}{$\begin{array}{l}\text { Tenax } \\
\text { carbone } \\
\text { graphité }\end{array}$} \\
\hline & & Q & S. $10^{7}$ & Q & S. $10^{7}$ & $Q$ & S. $10^{7}$ \\
\hline 17222,72 & octanoate de propyle & - & - & 59 & 0,39 & 96 & 0,58 \\
\hline 17322,83 & I-méthylnaphtalène & - & - & 49 & 1,09 & 45 & 1,87 \\
\hline$|7422,9|$ & tridécane & 96 & 3,28 & 94 & 1,70 & 93 & 2,97 \\
\hline 17523,62 & 2,5-dim éthylundécane & 87 & 3,27 & 50 & 1,62 & 87 & 3,36 \\
\hline 17624,17 & heptylcyclohexane & 89 & 0,39 & - & - & - & - \\
\hline | 7725,4 | & caprylate de butyle & 94 & 0,80 & 96 & 0,37 & 97 & 0,87 \\
\hline 17825,62 & décanoate d'éthyle & 64 & $|, 4|$ & 95 & 0,62 & 97 & 1,43 \\
\hline 17925,72 & tétradécane & 93 & 1,33 & 98 & 0,52 & 98 & 1,12 \\
\hline 18026,12 & junipène & 90 & 0,52 & - & - & 97 & 0,48 \\
\hline $18 \mid 26,47$ & caryophyllène & 99 & 0,95 & 95 & 0,49 & 99 & 1,13 \\
\hline 18227,39 & 4,6-diméthyldodécane & - & - & - & - & 87 & 0,32 \\
\hline 18328,38 & pentadécane & 93 & 0,61 & - & - & 93 & 0,63 \\
\hline
\end{tabular}

\section{Performances de fixation des COV et perfor- mances d'identification}

Les chiffres cités ci-après résultent de la combinaison de deux facteurs permettant de porter un jugement de valeur : aptitude de l'adsorbant à fixer des COV plus ou moins spécifiques d'une part et d'autre part, adéquation de la rampe thermique imposée à la colonne chromatographique puisque cette rampe définit une qualité de séparation, conditionnant elle même le caractère "incontestable" de l'identification. Or il convient de rappeler que cette rampe est commune à toutes nos analyses et il peut se trouver des coélutions qui auraient pu être, sinon évitées, tout au moins limitées au moyen d'une rampe plus adaptée, mais nous tenons à conserver des conditions opératoires simples, donc polyvalentes.

Compte tenu de cette remarque préalable ajoutée à nos critères d'identification définis plus haut nous pouvons prétendre que :

- I45 composés sont identifiés sur Tenax dont 102 avec $Q \geq 75$ et 70 avec $Q \geq 90$,

- $|4|$ composés sont identifiés sur Chromosorb dont 87 avec $Q \geq 75$ et 54 avec $Q \geq 90$,

- 147 composés sont identifiés sur Tenax-carbone graphité dont 105 avec $Q \geq 75$ et 68 avec $Q \geq 90$.

On peut donc admettre que les conditions analytiques imposées au chromatographe sont relativement acceptables.

\section{Degré de spécificité de fixation des COV, éta- blissement d'un diagnostic}

Si on accepte de considérer l'ensemble des trois tubes : I84 COV sont identifiés, autrement dit, lorsqu'un mélange très complexe doit être analysé de façon un peu exhaustive, la thermodésorption d'un ensemble de tubes doit être envisagée.

Par ailleurs il convient de noter que parmi ces 184 composés identifiés, 
34 le sont avec $\mathrm{Q} \geq 90$ sur les trois tubes,

20 avec $Q \geq 90$ sur 2 tubes,

25 avec $Q \geq 90$ sur un seul tube.

$\mathrm{Si}$ on accepte volontiers l'idée qu'un mélange très complexe de COV suppose une étude "multi-tubes", il convient néanmoins de porter un jugement sur le degré d'efficacité, ou d'erreur, commise par un opérateur qui utiliserait un seul lit de matériau adsorbant. En effet les performances de chaque tube paraissent proches en terme de nombre de composés identifiés $(145,14 \mid$ et 147).

En revanche la question qui importe est la suivante : estce qu'il existe au moins un tube, parmi ceux testés au cours de ce travail, qui présente une aptitude de fixation assez large pour attirer l'attention de l'opérateur sur la nécessité de compléter l'étude entreprise avec des tubes plus spécifiques afin d'éviter une erreur majeure? En effet le danger est d'avoir involontairement "occulté" une ou plusieurs familles de composés parce que tous les membres de cette famille sont ignorés par cet adsorbant.

Quelques éléments de réponse à cette question sont déjà connus :

- les produits très légers ne sont pas retenus sur les tubes testés ici ;

- les acides organiques ne sont pas retenus, mais les indices de leur présence sont là : nous avons identifié alcools et esters, l'attention de l'opérateur est donc sollicitée ;

- aucune amine n'a été détectée au cours de cette étude, ce qui paraît peu plausible. En fait, les amines en C2, C3 présentent un volume de perçage faible vis à vis d'adsorbants classiques et non spécifiques, ce qui peut conduire à une éviction artificielle de ces composés, surtout dans un milieu un peu chargé induisant des compétitions d'adsorption éventuellement sévères ;

- peu d'aldéhydes et cétones ont été identifiées mais elles le sont cependant en nombre suffisant pour alerter et mettre en place des moyens spécifiques.

Pour toutes ces familles chimiques, les matériaux adsorbants ou démarches analytiques très spécifiques sont connus :

- adsorption sur Spherocarb pour les produits très légers ;

- adsorption sur Porapak pour les acides organiques ;

- adsorption sur Porapak $N$ pour les amines ;

- adsorption sur silice greffée DNPH pour les aldéhydes et cétones.

Mais ces matériaux spécifiques imposent souvent une colonne chromatographique qui peut également être particulière, ou une démarche analytique qui l'est tout autant (par exemple LC-MS pour les aldéhydes et cétones). Ce type d'étude exhaustive sort du cadre que nous nous sommes fixés ici.

Le constat doit être dressé : la thermodésorption est une méthode analytique aisée à mettre en œuvre et plu- sieurs appareils performants existent sur le marché, mais il faut admettre que l'adsorbant universel est hors de portée. La question devient donc : quelle pourrait être la combinaison d'adsorbants qui permettrait d'établir un premier diagnostic fiable, ouvrant la voie à l'établissement d'une procédure analytique plus adaptée ? Cette étude permet de dégager quelques conclusions provisoires : le tube Tenax permet d'identifier 10 composés non reconnus par les autres tubes, le tube Chromosorb permet d'identifier II composés non reconnus par les autres tubes tandis que le tube Tenax/carbone graphité permet d'identifier seulement 6 produits non reconnus par les autres tubes. Ainsi nous confirmons des remarques déjà formulées auparavant : un tube dans lequel sont insérés des adsorbants "complémentaires" semble être le plus performant pour une analyse de type coup de sonde, par exemple : Tenax + Chromosorb.

\section{Extraction par solvant, quelle performance?}

Aux côtés de la thermodésorption, il existe une autre méthode officiellement reconnue et codifiée[6], c'est l'accumulation sur charbon actif suivie de l'extraction au moyen du sulfure de carbone.

Les avantages de cette méthode sont indéniables :

- l'extraction par solvant suivie d'une analyse GC/MS est une technique facile à mettre en œuvre puisqu'un "simple" GC/MS suffit ;

- il est facile de vérifier la non percée du matériau adsorbant, c'est le rôle dévolu à la deuxième partie aval du remplissage adsorbant ;

- les analyses chromatographiques peuvent être multipliées, avec en particulier des conditions différentes de split, très utiles dans le cas de gamme de concentrations inconnues.

Evidemment cette méthode présente également ses limites, dont la principale, imposée par la présence du solvant : tous les composés dont les temps de rétention sont voisins de celui du solvant seront occultés. Ceci concerne tous les composés légers dans le cas de $\mathrm{CS}_{2}$. Les résultats concernant notre étude comparative sont rassemblés dans le tableau II.

70 COV sont identifiés (avec $Q \geq 75$ ), dont 48 avec $Q$ $\geq 90$.

Parmi ces 70 composés, 47 COV ont été également identifiés par désorption thermique (voir tableau I).

Le fait marquant demeure celui-ci : $23 \mathrm{COV}$ ne sont identifiés que sur charbon actif, alors que selon les critères déjà décrits, ces composés ne l'ont pas été, tout au moins formellement, avec les thermodésorptions. On peut admettre que ces performances peuvent être attribuées au moins partiellement à la chaîne analytique (colonne de $60 \mathrm{~m}$ ) conduisant à une séparation plus performante, induisant une identification qui l'est tout autant.

En revanche, la limite de cette méthode est confirmée : 
la majorité des produits non détectés correspond aux produits légers qui éluent pendant le délai imposé par l'élution du solvant (solvant delay).

Quant aux produits lourds non détectés (produits plus lourds que le dodécane), c'est le paramètre durée d'analyse, volontairement limité à 50 minutes qui a été le facteur limitant (température de la colonne chromatographique limitée à $260^{\circ} \mathrm{C}$ ) pour tenter de ne pas trop s'éloigner de la durée moyenne des analyses issues de la désorption thermique dont la durée n'excède pas 30 minutes.

Tous les produits détectés avec $\mathrm{Q}>90$ dans les trois désorptions thermiques sont reconnus dans l'extraction liquide, sauf le 2,4-diméthylheptane, le I-éthyl-4-méthylcyclohexane, le 3-méthyldécane, le 2,6-diméthylundécane, le D-carvone.

Nous avons des facteurs de ressemblance très élevés $(97,98)$ pour certains produits identifiés, y compris pour des pics de surface faible. Ceci est probablement dû à une focalisation plus nette dans le cas d'une injection à la seringue ainsi qu'à une longueur de colonne nettement plus forte induisant une séparation plus poussée .

A condition de retenir une accumulation sur une superposition de lits adsorbants, par exemple ici Tenax+ Chromosorb, la thermodésorption est plus performante que l'extraction liquide (plus de 180 composés séparés et identifiés), en particulier pour la détection et l'identification des produits légers. En revanche le contrôle du non perçage est presque automatique sur une cartouche de charbon actif (il suffit de faire extraction et analyse des deux parties du tube) alors que le montage de deux tubes en série s'impose lorsqu'un prélèvement est conduit dans un milieu inconnu avec des tubes destinés à la thermodésorption.

\begin{tabular}{|c|c|c|c|c|c|}
\hline \multicolumn{6}{|c|}{$\begin{array}{c}\text { Tableau II : Résultats de l'analyse GC/MS après } \\
\text { accumulation sur charbon actif et extraction au } \\
\text { sulfure de carbone }\end{array}$} \\
\hline \multicolumn{6}{|c|}{$\begin{array}{cccc}\text { Produit } & \begin{array}{c}\text { Présent } \\
\text { sur tubes* }\end{array} & \text { Q } & \text { Surface. } 10^{7}\end{array}$} \\
\hline I & 12,28 & I-butanol & o*k & 91 & 2,105 \\
\hline 2 & 12,55 & benzène & o & 91 & 0,395 \\
\hline 3 & 12,68 & cyclohexane & $\mathrm{n}^{* * * *}$ & 90 & 0,129 \\
\hline 4 & 12,88 & 3-méthylhexane & $\mathrm{n}$ & 91 & 0,060 \\
\hline 5 & \multicolumn{2}{|c|}{ |3,67 |,2-diméthylcyclopentane } & $\mathrm{n}$ & 76 & 0,016 \\
\hline 6 & 13,82 & heptane & $\mathrm{n}$ & 94 & 0,161 \\
\hline 7 & 14,05 & trichloréthylène & o & 98 & 0,176 \\
\hline 8 & 14,66 & butanoate de méthyle & $\circ$ & 91 & 0,664 \\
\hline 9 & 15,18 & 3-méthyl I-butanol & $\circ$ & 90 & 0,155 \\
\hline 10 & 15,23 & méthylcyclohexane & $\circ$ & 97 & 0,102 \\
\hline II & 15,36 & 2-méthyl I-butanol & $\circ$ & 83 & 0,089 \\
\hline 12 & 16,00 & diméthyldisulfide & o & 97 & 7,23 \\
\hline 13 & 16,11 & Éthyl isobutanoate & $\circ$ & 90 & 0,212 \\
\hline 14 & 16,45 & I-pentanol & o & 90 & 0,170 \\
\hline
\end{tabular}

Tableau II : Résultats de l'analyse GC/MS après accumulation sur charbon actif et extraction au sulfure de carbone (suite)

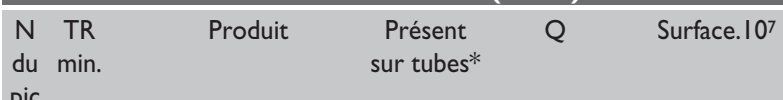

Pic

$\begin{array}{llllll}15 & 16,59 & \text { 4-méthylheptane } & \mathrm{n} & 76 & 0,023\end{array}$

$\begin{array}{llllll}16 & 16,92 & \text { toluène } & 0 & 91 & 1,077\end{array}$

$\begin{array}{lllll}17 \quad 17,82 & \text { butanoate d'éthyle } & 0 & 97 & 7,49\end{array}$

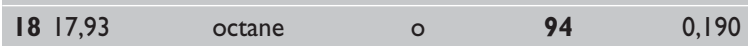

$19 \mid 8,07$ hexaméthylcyclotrisiloxane $0 \quad 86 \quad 0,020$

2018,15 propanoate de propyle $\quad 0 \quad 83 \quad 0,251$

$21 \quad 18,33$ acétate de butyle $\quad 0 \quad 78 \quad 0,114$

22 |8,6| tétrachloréthylène $\mathrm{n} \quad 97 \quad 0,130$

23 19,43 butanoate de I-méthyléthyle $\quad 0 \quad 83 \quad 0,098$

24 19,74propanoate de méthylpropyle $n \quad 78 \quad 0,169$

25 19,83butanoate de 2-méthyléthyle $\mathrm{n} \quad 95 \quad 0,200$

2620,05 butanoate de pentyle $n \quad 78 \quad 0,028$

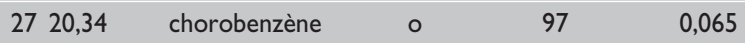

2820,49 4-méthyloctane $\quad \circ \quad 87 \quad 0,046$

2920,63 I,2,4-triméthylcyclohexane $\quad \circ \quad 9900,042$

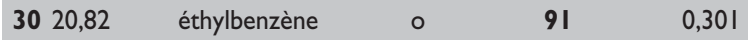

31 $21,15 \quad$ xylène $\quad 0 \quad 97 \quad 0,384$

$3221,21 \quad x y l e ̀ n e \quad 0 \quad 97 \quad 0,123$

$3321,61 \quad 1,2,4$-triméthylcyclohexane $\quad 0 \quad 93 \quad 0,029$

3421,70 butanoate de propyle $n \quad 90 \quad 0,972$

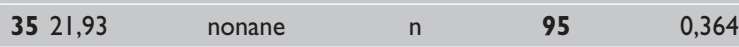

$\begin{array}{llllll}36 & 22,14 & \text { xylène } & n & 95 & 0,192\end{array}$

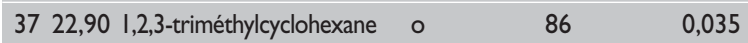

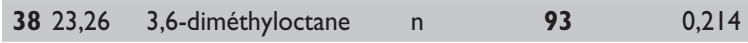

3923,66 propylcyclohexane $n \quad 92 \quad 0,199$

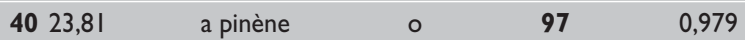

41 23,49 2-méthylnonane $\quad 0 \quad 93 \quad 0,135$

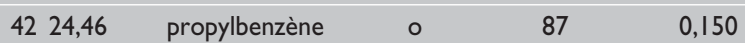

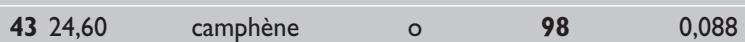

44 24,68 I-éthyl 2-méthylbenzène $\quad 0 \quad 95 \quad 0,443$

45 24,84 I-éthyl 2-méthylbenzène $\mathrm{n} \quad 94 \quad 0,167$

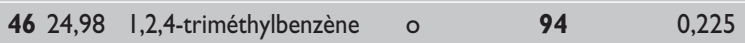

4725,22 b phéllandrène $\quad 0 \quad 91 \quad 0,220$

4825,39 butanoate de butyle $0 \quad 0860,921$

\begin{tabular}{|c|c|c|c|}
\hline 4925,47 & diméthyltrisulfure & ० & 91 \\
\hline & & 0 & \\
\hline
\end{tabular}

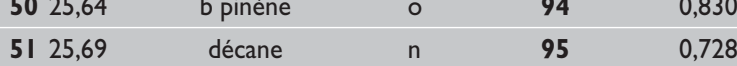

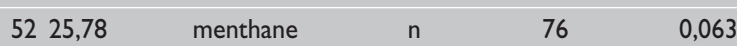

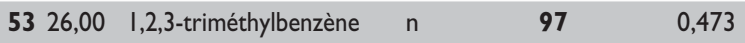

5426,49 4-méthyldécane $\quad 0 \quad 90 \quad 0,220$

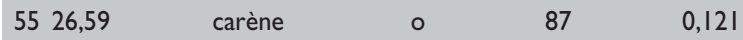

5626,81 p-isopropylméthylbenzène $0 \quad 81 \quad 0,062$

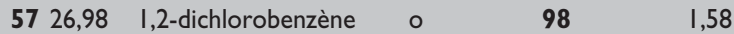

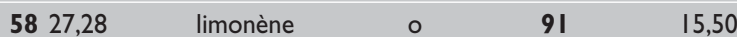

5927,42 butylcyclohexane $n \quad 81 \quad 0,242$

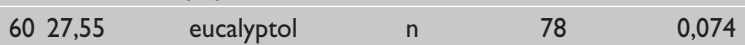

61 27,59butanoate de 3-méthylbutyle $\mathrm{n} \quad 78 \quad 0,069$

62 27,83 4-méthyldécane $\quad 0 \quad 91 \quad 0,132$

6328,12 I-éthyl 3,5-diméthylbenzène $\quad 0 \quad 95 \quad 0,068$ 


Tableau II : Résultats de Panalyse GC/MS après
accumulation sur charbon actif et extraction au
sulfure de carbone (suite)

\section{Comparaison des rapports de surfaces des pics majoritaires}

Bien que la quantification ne soit pas l'objet de cet article, un examen des rapports des surfaces obtenues pour ce qui touche aux composés majoritaires permet d'engager une réflexion sur les capacités de fixation par accumulation, et au-delà, sur la fiabilité de l'analyse quantitative qui, en principe, demeure l'objectif final de ces mesures. La comparaison des rapports de surface permet de situer la validité des concentrations déterminées au moyen de l'une ou l'autre des méthodes.

Ces résultats sont rassemblés dans le tableau III.

Premier constat encourageant : les composés définis comme étant majoritaires par leur surface le sont dans toutes les "filières analytiques".

Deuxième constat, mais évidemment sans réelle surprise : il apparaît une similitude entre l'accumulation sur Tenax et l'accumulation sur Tenax-carbone graphité, le rapport Tenax/Tenax-carbone graphité étant compris entre 0,944 et I,I 14 pour 6 produits sur 8 évalués. Sur ces huit rapports, 7 sont inférieurs à I. Compte tenu des conditions dans lesquelles les phases d'accumulation ont été conduites (en série) ces résultats illustrent la relative stabilité de l'émission collectée dans la canne métallique mais ne permettent pas de conclure que l'un des adsorbants est plus performant.

Par contre les comparaisons sont plus délicates avec le Chromosorb car la sélectivité de ce matériau adsorbant intervient, ce qui induit probablement un volume de perçage différent pour beaucoup de produits, y compris notre composé de référence le benzène. Il convient donc de cantonner ce type d'adsorbant à la quantification des composés polaires pour lesquels il a été développé.

La question majeure est évidemment relative à la comparaison charbon actif et adsorbant type Tenax puisque sans être totalement différents les rapports de surface peuvent présenter des écarts très conséquents. Ceci peut être dû à la variation avec le temps de l'émission des COV dans le casier pilote ou à des performances de

\begin{tabular}{|c|c|c|c|c|}
\hline \multicolumn{5}{|c|}{ Par rapport à un produit de référence } \\
\hline $\begin{array}{l}\text { Rapport de surface } \\
\text { des pics des produits }\end{array}$ & Charbon actif & Tenax & Chromosorb & $\begin{array}{l}\text { Tenax- } \\
\text { carbone } \\
\text { graphité }\end{array}$ \\
\hline toluène/benzène & 2,73 & 3,17 & 1,85 & 3,05 \\
\hline $\begin{array}{l}\text { trichloréthylène } \\
\text { /benzène }\end{array}$ & 0,446 & 0,95 & 0,444 & 1,00 \\
\hline $\begin{array}{l}\text { toluène } \\
\text { /trichloréthylène }\end{array}$ & 6,12 & 3,34 & 4,16 & 3,04 \\
\hline $\begin{array}{l}\text { éthylbenzène } \\
\text { /benzène }\end{array}$ & 0,762 & 0,898 & 0,942 & 1,01 \\
\hline$\alpha$ pinène/benzène & 2,48 & 1,455 & 2,33 & 3,69 \\
\hline $\begin{array}{l}\text { butanoate de } \\
\text { méthyle/benzène }\end{array}$ & 1,68 & 2,05 & 1,14 & 1,94 \\
\hline limonène/benzène & 39,2 & 46,0 & 29,8 & 45,0 \\
\hline $\begin{array}{l}\text { diméthyldisulfure } \\
\text { /benzène }\end{array}$ & 18,3 & 2,90 & 3,95 & 2,36 \\
\hline \multicolumn{5}{|c|}{ Par comparaison aux surfaces sur Tenax } \\
\hline Produit & \multicolumn{2}{|c|}{ Tenax/Chromosorb } & \multicolumn{2}{|c|}{$\begin{array}{l}\text { Tenax/ } \\
\text { Tenax-carbone graphite }\end{array}$} \\
\hline benzène & \multicolumn{2}{|l|}{0,872} & \multicolumn{2}{|l|}{0,923} \\
\hline trichloréthylène & \multicolumn{2}{|l|}{1,86} & \multicolumn{2}{|l|}{0,870} \\
\hline butanoate de méthyle & \multicolumn{2}{|l|}{1,57} & \multicolumn{2}{|l|}{0,981} \\
\hline diméthyldisulfure & \multicolumn{2}{|l|}{0,641} & \multicolumn{2}{|l|}{1,14} \\
\hline toluène & \multicolumn{2}{|l|}{1,49} & \multicolumn{2}{|l|}{0,958} \\
\hline éthylbenzène & \multicolumn{2}{|l|}{0,831} & \multicolumn{2}{|l|}{0,822} \\
\hline$\alpha$ pinène & \multicolumn{2}{|l|}{0,544} & \multicolumn{2}{|l|}{0,363} \\
\hline limonène & \multicolumn{2}{|l|}{1,346} & \multicolumn{2}{|l|}{0,944} \\
\hline
\end{tabular}

piégeage différentes des deux matériaux adsorbants.

Toutefois il apparaît au moins un composé, le limonène, qui paraît être fixé de manière comparable dans toutes les filières examinées ici.

Une des causes majeures de la dispersion des résultats est très probablement le perçage des adsorbants. Ce perçage a été évalué sur le charbon actif SKC. Dans le cas présent, la plupart des composés sont totalement adsorbés dans la première partie du tube. Mais le cyclohexane (10\%), le 3-méthyl hexane (10\%), l'heptane (14\%), et le décane $(3 \%)$ sont détectés de façon notable dans le culot de contrôle. Ces pourcentages sont calculés par le rapport des surfaces (rapportées à la surface de l'étalon interne) entre les deux parties du tube. Le perçage n'a pas été évalué sur les tubes destinés à la thermodésorption.

La deuxième raison de la dispersion relative des résultats présentés dans ce tableau est également due aux compétitions d'adsorption induites par la présence de composés en forte concentration. L'un des auteurs, Rodica Chiriac présente les concentrations déjà mesurées sur ce flux (tableau IV). Les concentrations sont très élevées, de l'ordre du $\mathrm{mg} . \mathrm{Nm}^{-3}$ pour la plupart des produits. Il a été vérifié[7] que ce ne sont pas forcément les composés présents majoritairement qui percent mais ils limitent la fixation de composés éventuellement 
minoritaires, ce qui pose le problème de l'étalonnage. Or la norme Afnor[5] relative à l'étalonnage semble ne pas prendre en compte cette compétitivité d'adsorption possible puisqu'elle repose sur l'addition de composés injectés à la seringue, à partir d'une solution liquide.

Pour l'étalonnage, il nous paraît plus sûr de conduire un étalonnage basé sur les techniques d'accumulation en phase gazeuse, soit à partir de mélanges commerciaux (comme le mélange EPA TOI4) très onéreux, ou à partir d'une source d'émission contrôlée [8] ou encore au moyen d'une rampe à perméation.

\begin{tabular}{|c|c|}
\hline Produit & Concentration en $\mu \mathrm{g} \cdot \mathrm{Nm}^{-3}$ \\
\hline benzène & 1660 \\
\hline heptane & 2340 \\
\hline trichloréthylène & 1505 \\
\hline toluène & 4232 \\
\hline octane & 302 \\
\hline tétrachloroéthylène & I 425 \\
\hline chlorobenzène & 293 \\
\hline éthylbenzène & 1092 \\
\hline m-xylène & 1519 \\
\hline p-xylène & 536 \\
\hline nonane & 686 \\
\hline o-xylène & 653 \\
\hline$\alpha$ pinène & 1511 \\
\hline mésitylène & 319 \\
\hline$\beta$ pinène & I 297 \\
\hline décane & 3893 \\
\hline limonène & 26748 \\
\hline undécane & I 873 \\
\hline dodécane & 346 \\
\hline
\end{tabular}

A l'évidence, si l'objectif visé d'une étude est la quantification, cette première approche de comparaison de méthodes analytiques rappelle que les paramètres majeurs demeurent le degré de fiabilité du contrôle des volumes de perçage et les compétitions d'adsorption qui renvoient à un étalonnage approprié.

\section{CONCLUSIONS}

Notre première conclusion est que le choix d'un tube dual Tenax TA-Spherocarb tel que préconisé par Environment Agency [3] permet certainement de suivre les composés définis comme prioritaires par cette agence, à condition toutefois que le milieu observé soit suffisamment dilué.

Nous avons nous-mêmes adopté une combinaison de plusieurs lits adsorbants permettant de couvrir l'essentiel des familles chimiques susceptibles d'être rencontrées. Ensuite il convient de mettre en œuvre des adsorbants plus spécifiques lorsqu'une étude exhaustive et quantitative est exigée. C'est ce qu'exige par exemple la compréhension de la cinétique de maturation d'un stoc- kage d'ordures ménagères en fonction des conditions de traitement, anaérobie ou non, et de stockage, en andain ou en silo (thèse en cours ${ }^{[9]}$ ).

La présente étude ne se prétend rien d'autre qu'être une première approche de comparaison de méthodes analytiques (avec les matériaux adsorbants associés): "voie thermodésorption - voie extraction par solvant". Les commentaires suscités nous invitent à poursuivre ce travail avec encore plus de rigueur (débits volumiques de prélèvement, contrôle des volumes de perçage) sans oublier toutefois que toute procédure développée sur le terrain comporte une part d'incertitude due à des difficultés propres à cet exercice.

Compte tenu des critères que nous nous étions imposés, il est toutefois possible d'admettre que du point de vue qualitatif, quelle que soit la méthode, les rendements en identification sont comparables, environ 200 composés sont identifiés. Les mesures effectuées montrent qu'en ce qui concerne la désorption thermique, la colonne chromatographique ordinaire, HP5MS, suffit pour la séparation et l'analyse qualitative des COV présents, mais il est évident que l'utilisation d'une colonne plus longue augmenterait encore les performances.

Du point de vue quantitatif, chaque méthode analytique permet d'établir une liste de composés classés dans l'ordre décroissant d'abondance. Ces listes présentent une composition qualitative d'une part, et un classement par abondance d'autre part, très semblables. Cet aspect est un premier point positif.

En revanche, cette étude met en lumière une certaine dispersion de résultats qui appelle une étude ultérieure si on rapporte les résultats à une référence interne, le benzène, (existant dans chaque mélange analysé), et ceci pour chaque filière. II conviendra de faire la part de ce qui relève de l'émission elle-même, des conditions de prélèvement (débits et volume de perçage) et de ce qui dépend éventuellement d'aspects plus fondamentaux (compétition d'adsorption liée aux concentrations induisant une variation du volume de perçage), induisant des techniques d'étalonnage adaptées en cours de validation.

\section{Rodica Chiriac}

Doctorante Ademe, Laboratoire d'application de la chimie à l'environnement, UCB Lyon I, 43, Bd. du II novembre 1918, 69622 Villeurbanne Cedex, France - Mail : Rodica.Chiriac@insa-lyon.fr

\section{Ludovic Fine}

Technicien CNRS, Département Analyse de gaz, Service central d'analyse CNRS, chemin du Canal, B. P. 22, 69390 Vernaison, France - Mail : I.fine@sca.cnrs.fr

Jean-Louis Gass

Ingénieur CNRS, Responsable du Service analyse de gaz, Service central d'analyse CNRS, chemin du Canal, B. P. 22, 69390 Vernaison, France - Mail : JL.GASS@sca.cnrs.fr

\section{Jean Carre}

Maître de conférences, Laboratoire d'application de la chimie à l'environnement, UCB Lyon I, 43, Bd. du III novembre 1918, 69622 Villeurbanne Cedex, France - Mail : Jean.Carre@insa-lyon.fr 
Nomenclature:

Temps de rétention

symbole: TR

unité : $\min$

Facteur de ressemblance

symbole : Q

sans unité

Surface de pic chromatogra-

phique

symbole: $S$

unité arbitraire "abondance.temps"

Concentration en phase gazeuse

symbole : [i]

unités : $\mu \mathrm{g} . \mathrm{Nm}^{-3}$ ou $\mathrm{mg} . \mathrm{Nm}^{-3}$

Concentration en phase liquide

symbole : [i]

unités : $\mathrm{mg}$.L-I

\section{Remerciements}

Nous tenons à remercier l'Ademe pour son aide par l'intermédiaire d'une bourse de thèse.

Nous remercions également l'équipe du Professeur Gourdon du LAEPSI/INSA qui nous a permis l'accès à ses pilotes d'OM dans le cadre d'une collaboration entre deux thèses Ademe.

\section{Bibliographie}

[I] Allen M. R., Braithwaite A., Hills C. C. Environ. Sci. Technol. I997, 3 I (4) |054-106 |

[2] Bradley A. D., Cook D. J., Edwards
J. S., Johnston A. G., Linforth R. S. T., Taylor A. J ; Sardinia 200I, Cagliari I-5 octobre $200 \mid$

[3] Environment Agency (2002) Investigation of the composition, emissions and effects of trace components in landfill gas. R\&D Project report PI438, Environment Agency, Bristol

[4] Environment Agency (2002) Guidance for monitoring trace components in landfill gas, Environment Agency, Bristol

[5] Norme NF EN ISO I60। 7-I mars 200I Echantillonnage et analyse des composés organiques volatils par tube à adsorption/désorption thermique/chromatographie en phase gazeuse sur capillaire

[6] Norme NF ISO 16200-I décembre 200 I Échantillonnage et analyse des composés organiques volatils par désorption au solvant/chromatographie en phase gazeuse

[7] Chiriac R. thèse Étude des émissions de composés organiques volatils par les centres d'enfouissement technique d'ordures ménagères. Evaluation de l'impact environnemental soutenance prévue à Lyon en septembre 2004

[8] Gass J. L., Cosic R., Ponchin G., Lasnel L. Procédé et dispositif de mélange de gaz. PCT Int. Appl. WO 03064016,2003

[9] Lornage R. thèse Évaluation des impacts environnementaux de trois filières de stockage d'ordures ménagères, en cours depuis septembre 2003

\begin{abstract}
Droits de propriété, économie et environnement

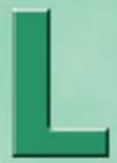
$\square$
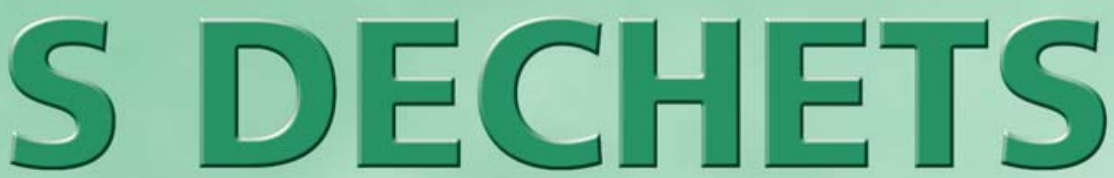

23-25 Juin 2004 - Aix-en-Provence

Les déchets posent aujourd'hui un des problèmes environnementaux redoutables qui à la différence d'autres domaines n'est pas en voie de solution. Tout ce passe comme si le recours aux solutions techniques cherchant à résoudre les effets masquait la recherche relative aux causes. La multiplication des réglementations publiques aboutit trop souvent à aggraver les problèmes ainsi qu'en témoignent les expériences françaises et étrangères. Face à la marée montante des déchets, les solutions réglementaires sont nécessaires mais insuffisantes. II importe aujourd'hui, comme pour les autres ressources environnementales, de considérer les instruments économiques et institutions juridiques permettant d'échapper à la "Tragedy of the Commons".
\end{abstract}

\title{
Constat et concepts
}

Statut juridique et incitations économiques.

L'approche réglementaire de la collecte et du traitement.

Evaluation et limites de la politique des déchets en France.

Evaluation critique des technologies propres.

Les acteurs économiques : producteurs de déchets ? Une

approche en termes de responsabilité.

Déchets et activité économique : comment concilier

compétitivité et Environnement.

\section{Expériences - Etudes de cas}

Gestion des déchets : expériences internationales.

La bourse des déchets : un outil adapté à la

politique de valorisation.

La valorisation de la fraction fermentescible des

ordures ménagères.

La protection des droits de propriété à travers la localisation des installations.

\section{Approche prospective de la gestion des déchets}

La répression des infractions au niveau international : influence sur les comportements. Le Principe Pollueur Payeur devrait-il être remplacé par le Principe Producteur Payeur? Incitations économiques en matière d'élimination.

Le marché des droits en matière de déchets : vers des permis transférables.

L'impact environnemental du libre marché dans le cadre de l'élargissement de l'Union Européenne.

Partenaire presse

ENWJBONNEMIENT 8 TECNNIOUE

Conférence organisée par I'International center for research on environmental issues (I.C.R.E.I) - Centre d'Analyse économique environnement (CA2E), Faculté d'économie appliquée Université d'Aix-Marseille III (F.E.A.)

Programme et enregistrement sur www.environnement-propriete.org 